\title{
FATORES ASSOCIADOS À COLONIZAÇÃO AXILAR POR MICRORGANISMO RESISTENTE EM PACIENTES NA UNIDADE DE TERAPIA INTENSIVA
}

\author{
FACTORS CONNECTED TO AXILLARY COLONIZATION BY RESISTANT ORGANISMS OF INTENSIVE \\ CARE UNIT PATIENTS
}

\author{
Rafaela Arcanjo ${ }^{a}$, Adriana Cristina de Oliveira ${ }^{b}$ \\ arafaela.aarcanjo@gmail.com, badrianacoliveira@gmail.com \\ Universidade Federal de Minas Gerais - Belo Horizonte (MG), Brasil
}

Data de recebimento do artigo: 29/02/2016

Data de aceite do artigo: 14/10/2016

\section{RESUMO}

Introduçáo: A disseminação da resistência bacteriana quase sempre está vinculada a pacientes colonizados ou infectados. A deteç̧áo de pacientes colonizados permite a implementaçáo oportuna de intervençóes destinadas a prevenir a transmissáo de microrganismos resistentes e consequentemente a infecçáo. Objetivo: Analisar a incidência de colonização axilar por microrganismo resistente (MR) e os possíveis fatores de risco associados, em pacientes internados em uma Unidade de Terapia Intensiva (UTI). Materiais e métodos: Tratou-se de uma coorte histórica realizada entre agosto de 2013 a agosto de 2015, envolvendo 837 pacientes. Os dados foram coletados de registros dos prontuários dos pacientes e resultados de exames microbiológicos. Resultados e conclusóes: Dentre os pacientes avaliados, 122 $(14,6 \%)$ estavam colonizados por MR, destacando-se: Enterobacter sp. (25\%), Acinetobacter baumannii (20\%), Staphylococcus aureus (15\%), Pseudomonas aeruginosa (12\%). Os fatores associados à colonização por microrganismos resistentes foram: tempo de permanência na $U T I \geq 9(\mathrm{OR}=32,4)$, uso de procedimento invasivo $(\mathrm{OR}=25,7)$ e uso de antimicrobiano $(\mathrm{OR}=13,9)$. A relaçáo entre colonizaçáo por microrganismo resistente e os fatores de risco evidencia a necessidade de monitorização e instituição de protocolos que visem a adesão às medidas de controle da disseminaçáo desses microrganismos.

Palavras-chave: Controle de infecçóes; resistência microbiana a medicamentos; segurança do paciente; Unidade de Terapia Intensiva; susceptibilidade antimicrobiana.

\section{ABSTRACT}

Introduction: The spread of bacterial resistance is almost always linked to colonized or infected patients. The detection of patients colonized allows timely implementation of interventions to prevent transmission of resistant organisms and consequently infection. Objective: To analyze incidence colonization by resistant microorganism (MR) in the axillary region and possible risk factors in patients admitted to an Intensive Care Unit (ICU). Materials and methods: It is about a historical cohort study conducted between august 2013 and august 2015, involving 837 patients. The data were collected from the medical records of patients and results of microbiological tests. Results and conclusions: Among the patients studied, $122(14.6 \%)$ were colonized by MR, especially: Enterobacter sp. 25\%, Acinetobacter baumannii 20\%, Staphylococcus aureus $15 \%$, Pseudomonas aeruginosa $12 \%$. The factors associated with colonization by resistant microorganisms were: length of stay in ICU $\geq 9(\mathrm{OR}=32.4)$, use of invasive procedure $(\mathrm{OR}=$ 25.7) and the use of antimicrobial $(\mathrm{OR}=13.9)$. The relationship between colonization by MR and risk factors shows the need for monitoring and protocol institution aimed at accession to the control measures of the spread of these microorganisms.

Keywords: Control infections; microbial drug resistance; patient safety; Intensive Care Unit; antimicrobial susceptibility. 


\section{Introdução}

As infecçóes relacionadas à assistência a saúde (IRAS), causadas por microrganismos resistentes (MR), são cada vez mais frequentes em hospitais, em especial nas unidades de terapia intensiva (UTI). A gravidade e a extensão das doenças causadas pela multirresistência, entre as espécies bacterianas, constituem um importante problema mundial, representando grande ameaça para a segurança do paciente $e^{1,2,3}$.

Um aspecto que tem sido apontado como facilitador para ocorrência de IRAS aumentando as possibilidades de complicações e até mesmo do óbito entre pacientes críticos se refere à colonização destes ${ }^{4,5}$.

A colonização de pele e mucosas, por MR, de pacientes hospitalizados tem merecido crescente atenção dos serviços de saúde, mas não há ainda estimativas do impacto mundial dessas colonizaçóes ${ }^{5}$, sabe-se que a identificação de indivíduos colonizados, mesmo quando esses não apresentam sinais de infecção ativa, contribui para a redução da circulação desse agente e reduz sua participação na etiologia das IRAS ${ }^{6}$.

Para a contenção da disseminação de bactérias resistentes, medidas como cultura de vigilância e isolamento de pacientes portadores desses microrganismos são destacadas em diversos estudos ${ }^{7-10}$.

Há evidências de colonização em estudos para vários sítios de recuperação desses microrganismos, sendo os de maior incidência: a região nasal com colonização entre 58 e $91 \%$ dos pacientes, com destaque para as bactérias gram-positivas em especial ao Staphylococcus aureus resistentes à oxacilina (MRSA) e também a região perianal (de 26 a 63\%) com maior colonização por bactérias gram-negativas, sendo os MR mais relatados Pseudomonas aeruginosa, Acinetobacter, Escherichia coli e Enterobacter $s p p^{5,9,11,12}$

A região axilar ainda é pouco utilizada nos estudos para detecção de MR, mas há indícios de que a colonização nessa regiáo possa variar de 16 a $32 \%$ entre os pacientes na UTI, constituindo assim uma fonte de microrganismos, muitas vezes, subestimada nos estabelecimentos de cuidados em saúde ${ }^{6-8}$. Há assim a necessidade de estudos direcionados a monitorização de várias partes do corpo, destacando-se as regióes sebáceas e úmidas, onde há maior facilidade de proliferação desses microrganismos ${ }^{5,8,12}$.

A relevância deste estudo justifica-se pela escassez de publicações encontradas sobre a topografia de colonização da região axilar, analisando assim sua incidência e os possíveis fatores de risco associados, em pacientes internados em uma Unidade de Terapia Intensiva.

Espera-se que este estudo possa subsidiar futuras reflexôes sobre a importância da identificação precoce de pacientes colonizados de forma a minimizar a transmissão cruzada de microrganismos e permitir um melhor planejamento multiprofissional favorecendo a construção de uma prática assistencial mais segura ao paciente.

\section{Métodos}

Tratou-se de um estudo epidemiológico do tipo coorte prospectivo realizada em uma Unidade de Terapia Intensiva para adultos de um hospital geral, de médio porte do interior do estado de Minas Gerais. Foram incluídos no estudo todos os pacientes admitidos na UTI no período entre agosto de 2013 a agosto de 2015 submetidos ao protocolo de cultura de vigilância da instituição para microrganismos resistentes.

A coleta de material para cultura de vigilância foi realizada rotineiramente na unidade de estudo, por profissionais de enfermagem treinados para essa prática. A unidade conta com protocolo instituído de coleta de swab axilar, para todos os pacientes admitidos que se encaixassem nos seguintes critérios de admissão: admissão por transferência de outra unidade hospitalar, história de internação prévia em UTI de até um ano e pacientes institucionalizados (asilares, casa de repouso, presidiários etc.).

Uma vez admitidos na UTI e enquadrados no protocolo de cultura de vigilância, pelos critérios de inclusão já descritos, estes eram, então, submetidos à nova coleta de culturas realizadas semanalmente até a alta da UTI ou óbito.

Para os pacientes que não se enquadraram nos critérios de inclusão da admissão, após permanecerem na unidade por sete dias, foram coletadas culturas de vigilância, sendo nesses pacientes realizadas novas coletas de acompanhamento semanalmente até sua alta ou óbito.

Para definição dos marcadores de resistência bacteriana, adotados neste trabalho, seguiu-se o protocolo estabelecido pela Comissão de Controle de Infecção Hospitalar da instituição de estudo, em consonância com o Clinical and Laboratory Standards Institute (CLSI). Foram considerados Staphylococcus aureus quando resistentes à oxacilina (análogo da meticilina no Brasil); Enterococcus sp., resistentes à vancomicina (VRE) e teicoplanina; Streptococcus pneumoniae resistentes à vancomicina; Pseudomonas sp., resistentes ao imipenem e/ou meropenem; enterobacteriáceas (Klebsiella, Serratia, Enterobacter, Escherichia coli, Proteus), resistentes a aminoglicosídeos, cefalosporinas de $3^{\text {a }}$ geração e fluorquinolonas ${ }^{13}$.

A coleta dos dados foi realizada diariamente pela pesquisadora, a vigilância epidemiológica e o 
monitoramento da evolução do paciente foram realizados diariamente. Adotou-se um instrumento de coleta de dados dos pacientes, tais como: identificação; idade; tempo de permanência na UTI; colonização por MR; uso de procedimentos invasivos (ventilação mecânica, cateteres vesical de demora e venoso central); IRAS conforme o critério do National Healthcare Safety Network (NHSN) ${ }^{14}$; uso de antimicrobiano e desfecho. Os dados foram obtidos dos registros de enfermagem, dos prontuários dos pacientes e dos resultados de exames microbiológicos. Os dados foram tabulados no programa estatístico Statistical Package for the Social Sciences (SPSS) para Windows versão 19.0 .

Primeiramente, foi realizada a análise descritiva dos dados para caracterizar a população de estudo, com a apresentação dos valores das medianas das variáveis contínuas, uma vez que as variáveis contínuas não apresentaram distribuição normal, e do valor absoluto e percentual das variáveis categóricas. Para testar a normalidade das variáveis, utilizou-se o teste Kolmogorov-Smirnov.

A análise estatística foi realizada pelo teste $\mathrm{T}$ de Student e teste Chi-Quadrado. A análise bivariada foi utilizada para identificar as variáveis com $\mathrm{p}<0,20$ para inserção destas em modelo multivariado.

As variáveis associadas à colonização de pacientes com $\mathrm{p}<0,20$ foram inseridas no modelo de regressão logística. Foi considerado um nível de significância de 5\% e intervalo de confiança de $95 \%$.

Atendendo à Resolução no 466/2012 do Conselho Nacional de Saúde, este estudo foi submetido ao Comitê de Ética em Pesquisa (COEP) da Universidade Federal de Minas Gerais (UFMG), tendo sido aprovado, conforme parecer CAAE - 25496113.1.0000.5149, e, ainda, por se tratar de um estudo realizado no âmbito do Hospital São João Batista (Viçosa/MG), também foi encaminhado à Comissão de Ensino, Pesquisa e Extensão (CEPE), tendo recebido aprovação.

\section{Resultados}

\section{Características gerais dos pacientes admitidos na UTI}

Durante o período de estudo, foram acompanhados 837 pacientes, sendo $472(56,4 \%)$ do sexo masculino, com idade média de 72,3 anos e média de permanência de 7,8 dias na UTI. A maioria era paciente clínico $(75,5 \%)$, proveniente da comunidade $(82 \%)$, transferidos de outro hospital $(15,8 \%)$ e institucionalizados $(2,2 \%)$, conforme apresentado na Tabela 1 .
Tabela 1: Características gerais dos pacientes $(\mathrm{n}=837)$ acompanhados, de acordo com as variáveis coletadas na UTI (Viçosa, 2015).

\begin{tabular}{lcc} 
Variáveis & No & $\%$ \\
\hline Sexo & & \\
\hline Masculino & 472 & 56,4 \\
\hline Feminino & 365 & 43,6 \\
\hline Idade (média em anos) & 72,3 & - \\
\hline Origem & & \\
\hline Comunidade & 686 & 82,0 \\
\hline Outro hospital & 132 & 15,8 \\
\hline Institucionalizados & 19 & 2,2 \\
\hline Infecçáo comunitária & & \\
\hline Sim & 376 & 45,0 \\
\hline Não & 461 & 55,0 \\
\hline Permanência na UTI (média em dias) & 7,8 & - \\
\hline Procedimento invasivo & & \\
\hline Sim & 569 & 68,0 \\
\hline Não & 268 & 32,0 \\
\hline Uso de antimicrobiano & & \\
\hline Sim & 544 & 65,0 \\
\hline Não & 293 \\
\hline Infecçóes na UTI & & 35,0 \\
\hline Sim & 209 & 25,0 \\
\hline Não & 628 & 75,0 \\
\hline Desfecho & & \\
\hline Alta & 616 & 73,6 \\
\hline Óbito & 188 & 22,5 \\
\hline Transferência hospitalar & 33,9 \\
\hline & & \\
\hline
\end{tabular}

Dos 837 pacientes, 569 (68\%) utilizaram algum tipo de procedimento invasivo, assim distribuídos: 256 (45\%) cateter venoso central, $502(88,2 \%)$ cateter vesical de demora e 375 (66\%) ventilação mecânica. Vale ressaltar que um paciente pode ter sido submetido a mais de um procedimento invasivo.

Quanto ao desfecho dos 837 pacientes, $616(73,6 \%)$ foram transferidos para outros setores do hospital, e, desses, 135 (22\%) faleceram, $473(76,8 \%)$ tiveram alta hospitalar e $8(1,3 \%)$ foram transferidos para outro hospital.

Dentre os pacientes que faleceram, verificou-se que a permanência média deles antes do óbito variou de 9 a 35 dias.

\section{Pacientes com cultura axilar positiva para colonização por MR}

Foi realizado o total de 2.160 culturas de swab axilar para os 837 pacientes avaliados, destas 1.852 culturas eram negativas para MR referentes aos $715(85,4 \%)$ pacientes não colonizados e foram avaliadas 308 culturas positivas, para os $122(14,6 \%)$ pacientes colonizados por MR. 
A média e o número médio de culturas de cada paciente foram de 2,0 e 3,0, respectivamente.

Dentre os microrganismos resistentes isolados nas 308 culturas, de swab axilar, destacaram-se: Enterobacter sp. (25\%), Acinetobacter baumannii (20\%), Staphylococcus aureus (15\%), Pseudomonas aeruginosa (12\%), Proteus $(6 \%)$, Enterococcus (10\%) e outros (12\%).

$\mathrm{Na}$ colonização, também foram detectados VRE, Klebsiella pneumoniae produtoras de betalactamases de espectro ampliado (ESBL), Klebsiella pneumoniae MR/ ESBL, Klebsiella pneumoniae resistente aos carbapenêmicos (KPC), cada um destes representando 3,7\%.

Os fatores de risco para a colonização por microrganismo resistente foram: tempo de permanência na UTI, procedência do setor de emergência e especialidade clínica na admissão, uso de antimicrobiano, uso de procedimento invasivo e infecção na UTI, sendo estatisticamente significativos para $\mathrm{p}<0,05$ (Tabela 2).

Tabela 2: Distribuição dos pacientes colonizados ( $n=122)$ e não colonizados $(n=715)$ de acordo com as variáveis do estudo na Unidade de Terapia Intensiva (Viçosa, 2015).

\begin{tabular}{|c|c|c|c|c|}
\hline \multirow{3}{*}{$\begin{array}{l}\text { Variáveis } \\
\text { Sexo }\end{array}$} & \multirow{2}{*}{$\begin{array}{l}\text { Categoria } \\
\text { Feminino }\end{array}$} & \multirow{2}{*}{$\begin{array}{l}\text { Náo colonizados } \\
\qquad \begin{array}{c}\mathbf{N}(\%) \\
50(41)\end{array}\end{array}$} & \multicolumn{2}{|c|}{$\begin{array}{l}\text { Valor } \mathrm{p}^{*} \\
\mathrm{~N}(\%)\end{array}$} \\
\hline & & & $300(42)$ & $>0,32$ \\
\hline & Masculino & $72(59)$ & $415(58)$ & \\
\hline Idade & Mediana em anos & 61 & 67 & $>0,26$ \\
\hline Procedência & $\begin{array}{l}\text { Outros setores } \\
\text { Emergência } \\
\text { Outros hospitais }\end{array}$ & $\begin{array}{l}43(35) \\
65(53) \\
14(12)\end{array}$ & $\begin{array}{c}256(36) \\
405(57) \\
51(7)\end{array}$ & $<0,04$ \\
\hline Permanência na UTI & $\begin{array}{l}1-4 \\
5-8 \\
\geq 9\end{array}$ & $\begin{array}{c}33(27) \\
26(21,3) \\
63(51,7)\end{array}$ & $\begin{array}{l}400(56) \\
200(28) \\
115(16)\end{array}$ & $<0,02$ \\
\hline $\begin{array}{l}\text { Especialidade clínica } \\
\text { na admissáo }\end{array}$ & $\begin{array}{l}\text { Clínica } \\
\text { Cirúrgica } \\
\text { Trauma }\end{array}$ & $\begin{array}{c}95(77,9) \\
22(18) \\
5(4,1)\end{array}$ & $\begin{array}{c}517(72,3) \\
166(23,2) \\
32(4,5)\end{array}$ & $<0,01$ \\
\hline Uso de antimicrobiano & $\begin{array}{l}\text { Sim } \\
\text { Não }\end{array}$ & $\begin{array}{l}88(72) \\
34(28)\end{array}$ & $\begin{array}{l}456(63,8) \\
259(36,2)\end{array}$ & $<0,03$ \\
\hline Procedimento invasivo & $\begin{array}{l}\text { Sim } \\
\text { Náo }\end{array}$ & $\begin{array}{l}102(83,6) \\
20(16,4)\end{array}$ & $\begin{array}{l}467(65,3) \\
248(34,7)\end{array}$ & $<0,02$ \\
\hline Infecção comunitária & $\begin{array}{l}\text { Sim } \\
\text { Não }\end{array}$ & $\begin{array}{l}28(23,0) \\
94(77)\end{array}$ & $\begin{array}{l}348(48,7) \\
367(51,3)\end{array}$ & $>0,47$ \\
\hline Infecção na UTI & $\begin{array}{l}\text { Sim } \\
\text { Não }\end{array}$ & $\begin{array}{l}83(68) \\
39(32)\end{array}$ & $\begin{array}{l}126(17,6) \\
589(82,4)\end{array}$ & $<0,02$ \\
\hline Desfecho & $\begin{array}{l}\text { Alta } \\
\text { Óbito }\end{array}$ & $\begin{array}{l}87(71,3) \\
35(28,7)\end{array}$ & $\begin{array}{l}534(75) \\
178(25)\end{array}$ & $>0,32$ \\
\hline
\end{tabular}

*Valor de p para o teste Chi-Quadrado de Pearson para variáveis categóricas e teste t para variáveis contínuas.

Dentre os pacientes que apresentaram infecçóes relacionadas ao cuidar em saúde, a idade média foi de 65 anos, $67 \%$ eram do sexo masculino e $86 \%$ utilizaram procedimentos invasivos.

$\mathrm{Na}$ análise bivariada, relacionou-se separadamente a variável colonização axilar por MR com: procedência, tempo de permanência na UTI, desenvolvimento de infecção relacionada ao cuidar em saúde, uso de procedimentos invasivos, especialidade na admissão e uso de antimicrobiano. Somente o tempo de $\geq 9$ dias $(\mathrm{OR}=8,06)$, o desenvolvimento de IRAS $(\mathrm{OR}=7,52)$, o uso de procedimentos invasivos $(\mathrm{OR}=9,67)$ e o uso de antimicrobiano $(\mathrm{OR}=5,43)$ foram estatisticamente significantes para o desenvolvimento de colonização axilar por $M R(p<0,01)$.

$\mathrm{Na}$ análise multivariada, o tempo de permanência de $\geq 9$ dias $(\mathrm{OR}=32,4)$, o uso de antimicrobiano $(\mathrm{OR}=13,9)$ e o uso de procedimento invasivo $(\mathrm{OR}=25,7)$ apresentaram significância estatística, mantendo-se com um fator de risco para o desenvolvimento de colonização por MR.

Observou-se também que 26,2\% (32/122) dos pacientes apresentaram colonização por mais de um microrganismo resistente durante o período de internação, sendo que $23 \%(28 / 122)$ desses pacientes tiveram as positividades nas culturas para mais de $02 \mathrm{MR}$ a partir da terceira cultura de vigilância, ou seja, após 21 dias de internação na unidade. 


\section{Discussão}

A maioria dos estudos que avaliaram a resistência aos antibióticos foi centrada em um único microrganismo resistente. Este estudo teve a intenção de definir aqueles pacientes com maior probabilidade de serem portadores de microrganismos resistentes, em uma parte do corpo. Isso se justifica quando se observa que os fatores de risco para resistência são compartilhados por várias espécies bacterianas ${ }^{15-18}$.

Na pele, em locais parcialmente ocluídos, a exemplo da prega inguinal e região axilar, as populaçóes bacterianas podem ser mais estáveis ao longo do tempo. Em contrapartida, nos locais mais secos e mais expostos, a diversidade microbiana pode ser mais elevada e com maior oscilação temporal. As alteraçōes podem ocorrer após alguns fatores externos, como o uso de antibióticos ou a aquisição de algum patógeno ${ }^{19,20}$.

$\mathrm{Na}$ avaliação da microbiota de populaçóes saudáveis, a mucosa nasal e a virilha foram identificadas entre os pontos mais consistentes em relação à composição e à estrutura da comunidade microbiana em um estudo de caracterização da variação temporal da microbiota ${ }^{21}$.

Estudos demonstraram que a recuperação de microrganismos resistentes na região axilar, através de culturas de vigilância, apresenta prevalência de 16 a 32\%, neste estudo, para pesquisa de microrganismo de importância epidemiológica na região axilar, foi encontrado taxa menor do que nos estudos descritos ${ }^{5,12}$.

Os microrganismos de relevância epidemiológica com maior incidência identificados na colonização de pacientes na UTI avaliada foram: os bastonetes gram-negativos: Enterobacter sp. (25\%), Acinetobacter baumannii (20\%) e Pseudomonas aeruginosa (12\%).

Os bacilos gram-negativos são amplamente descritos na literatura como um dos principais microrganismos causadores de colonização e infecção nas instituiçóes hospitalares. Vários estudos têm relatado a presença desse microrganismo, com cepas resistentes, presentes na pele de pacientes hospitalizados em UTI, com destaque para recuperação desses microrganismos nas regióes de virilha e em poucos estudos relatando a presença na axila ${ }^{20,21,23,24}$.

Dentre os cocos gram-positivos destacaram-se os Enterococcus faecium (10\%) resistentes à vancomicina e Staphylococcus aureus (15\%) resistente a oxacilina. Perfil similares de colonizaçáo por espécies bacterianas de relevância epidemiológica tem sido verificado em segmentos de pacientes em instituiçấo de longa permanência para vários sítios de colonizaçãó ${ }^{17,20,22}$.

Ressalta-se que nos registros de estudos com pacientes colonizados por Staphylococcus aureus, a prevalência desse microrganismo é mais evidenciada naqueles pacientes com fatores predisponentes como: hospitalização prolongada, procedimentos cirúrgicos e uso de dispositivos invasivos ${ }^{10,12,22}$.

$\mathrm{Na}$ colonização por VRE, neste estudo destacou-se o Enterococcus faecium, igualmente ao registrado em estudos internacionais, nos quais verificou-se a predominância do mesmo microrganismo, porém mais comumente encontrado em estudos com recuperação por swab na regiáo retal, sendo registrado para essa espécie multirresistência a vários antibióticos, além de apresentar a aquisiçáo de resistência nos últimos anos à vancomicina, linezolida e daptomicina ${ }^{25,26}$.

Verificou-se que pacientes com permanência maior ou igual a nove dias na unidade possuem risco 32 vezes maior $(\mathrm{OR}=32,4)$ quando comparado aos grupos que permaneceram menos que nove dias, resultado este que se encontra em consonância com os achados de outros estudos, que evidenciaram o tempo de permanência hospitalar em UTI como fator predisponente à aquisição de $\mathrm{MR}$, uma vez que as UTIs são locais que concentram os pacientes clínicos e cirúrgicos mais graves, o que leva a amplo uso de antimicrobianos, um dos fatores contribuintes para aumento da resistência bacteriana ${ }^{7,8,16}$.

$\mathrm{O}$ uso de antimicrobiano durante o período de permanência apresentou risco quase catorze vezes maior para colonização por MR. A importância do uso de antimicrobianos e a colonização por bactérias resistentes verificada neste estudo podem estar relacionadas às alteraçóes ocorridas na microbiota após terapia antimicrobiana. $\mathrm{O}$ uso prévio de antimicrobianos tem sido identificado como um importante fator de risco para a aquisição de microrganismos resistentes. Estudos relataram que as principais características relacionadas à colonização por gram-positivo, em especial ao MRSA, foram o uso de fluoroquinolonas e glicopeptídeo de forma inapropriada e sem critério, e na colonização por VRE, destacou-se o uso de glicopeptídeo prévio ${ }^{12,18,21,24}$.

$\mathrm{O}$ uso dos antimicrobianos de uma maneira maciça e indiscriminada exige medidas urgentes para combater o surgimento de novas cepas bacterianas resistentes, inclusive aos medicamentos antimicrobianos recentemente comercializados, levando a consequências importantes, com efeitos diretos na problemática das infecçóes hospitalares ${ }^{23,25}$.

O procedimento invasivo foi considerado como fatores associados ao risco de colonização axilar por bactérias resistentes, quase 26 vezes maiores que o risco de pacientes que não haviam sido submetidos a procedimentos invasivos. Em relação ao uso de procedimentos invasivos, estudos evidenciaram os cateteres endovenosos como um dos fatores de risco mais frequentes para a colonização por $\mathrm{MR}^{17,20,23}$.

A permanência de um patógeno como colonizador pode implicar a possibilidade de desfecho de infecção, dependendo das condiçóes imunológicas do paciente, e de 
associação com outros fatores preditivos das infecçôes, devido a aspectos do microrganismo, como virulência e carga microbiana, ou da resposta imune do hospedeiro ${ }^{17,20,21}$.

Estudos têm demonstrado que um dos maiores problemas relacionados ao cuidado inseguro em hospitais são ocasionados pelo aumento da propagação de MR no ambiente hospitalar e sua repercussão no desenvolvimento de IRAS ${ }^{23,25,26}$. Uma pesquisa que mensurou a prevalência desses eventos em hospitais de cinco países da América Latina mostrou que 37,1\% (501/1349) dos eventos ocorreram devido às IRAS causadas por $\mathrm{MR}^{26}$.

Múltiplos fatores têm sido descritos como possíveis predisponentes à colonização de pacientes na UTI como: idade avançada, tempo de permanência hospitalar, gravidade da doença de base, exposição a procedimentos invasivos, utilização de antimicrobiano e transferência entre hospitais e unidades ${ }^{17,20,25}$.

O controle efetivo de microrganismo resistente baseia-se em um conjunto de medidas de adesão estrita aos princípios básicos do controle da infecção como: a higiene das mãos, a identificação precoce e $\mathrm{o}$ isolamento de pacientes colonizados. $\mathrm{O}$ conhecimento dos fatores de risco e da epidemiologia local desses microrganismos ajuda a aprimorar o cumprimento das recomendaçóes.

Observa-se a necessidade de medidas para identificação de indivíduos portadores de $\mathrm{MR}$, na regiáo axilar e em diferentes partes do corpo, a fim de realizar controle, redução e prevenção da disseminação de microrganismos resistentes e o direcionamento da sistematização de condutas que previnam infecçóes relacionadas à assistência a saúde, com o objetivo de oferecer uma assistência segura no ambiente hospitalar.

\section{Contribuições das autoras}

Arcanjo RA e Oliveira AC participaram da concepção e delineamento do estudo, análise e interpretação dos dados, redação e revisão crítica do conteúdo intelectual do manuscrito. Todas as autoras aprovaram a versão final do manuscrito e são responsáveis por todos os aspectos do trabalho, incluindo a garantia de sua precisão e integralidade.

\section{Referências}

1. Minhas P, Perl TM, Carroll KC, Shepard JW, Shangraw KA, Fellerman D, et al. Risk factors for positive admission surveillance cultures for methicillin-resistant Staphylococcus aureus and vancomycin-resistant enterococci in a neurocritical care unit. Crit Care Med. 2011;39(10):2322-9.

2. Zarb P, Coignard B, Griskeviciene J, Muller A, Vankerckhoven V, Weist K, et al. The European Centre for Disease Prevention and Control (ECDC) pilot point prevalence survey of healthcare-associated infections and antimicrobial use. Eurosurveillance [Internet]. 2012 [citado em 2015 set. 15];17(46). Disponível em: http://bit. ly/2oZfWnO

3. Nascimento PVFS, Silva LGDM, Madureira PR. Fatores preditores para infecção ou colonizaçáo por bactérias multidroga resistentes: um estudo de caso-controle. Am J Infect Control. 2013;2(2):117-23.

4. Lee CC, Lee CH, Hong MY. Risk factors and outcome of Pseudomonas aeruginosa bacteremia among adults visiting the ED. Am J Emerg Med. 2012;30(6):852-60.

5. Chen YE, Tsao H. The skin microbiome: current perspectives and future challenges. J Am Acad Dermatol. 2013;69(1):143-55.

6. Thurlow CJ, Prabaker K, Lin MY, Lolans K, Weinstein RA, Hayden MK. Anatomic sites of patient colonization and environmental contamination with Klebsiella pneumoniae carbapenemase-producing Enterobacteriaceae at long-term acute care hospitals. Infect Control Hosp Epidemiol. 2013;34(1):56-61.

7. Birgand G, Lefevre LA, Lolom I, Ruppe E, Andremont A, Lucet JC. Duration of colonization by extended-spectrum b-lactamase-producing Enterobacteriaceae after hospital discharge. Am J Infect Control. 2013;41(5):443-7.

8. Marshall C, Richards M, McBryde E. Do active surveillance and contact precautions reduce MRSA acquisition? A prospective interrupted time series. PLos One. 2013;8(3):1-9.

9. Vikram HR, Dumiga DG, Kohan C, Havill NL, Tauman A, Boyce JM. Discontinuation of contact precautions for patients no longer colonized with methicillin-resistant Staphylococcus aureus. Infect Control Hosp Epidemiol. 2010;31(5):541-3.

10. Martinez-Capolino C, Reyes K, Johnson L, Sullivan J, Samuel L, Digiovine B, et al. Impact of active surveillance on methicillin resistant Staphylococcus aureus transmission and hospital resource utilization. J Hosp Infect. 2010;74(3):232-7.

11. Khanal R, Sah P, Lamichhane P, Lamsal A, Upadhaya $S$, Pahwa VK. Nasal carriage of methicillin resistant Staphylococcus aureus among healthcare workers at a tertiary care hospital in Western Nepal. Antimicrob Resist Infect Control. 2015;39(4):1-5.

12. Mermel LA, Cartony JM, Covington P, Maxey G, Morse D. Methicillin-Resistant Staphylococcus aureus colonization at different body sites: A prospective, quantitative analysis. J Clin Microbiol. 2011;49(3):1119-21.

13. Clinical and Laboratory Standards Institute (CLSI). Performance standards for antimicrobial disk susceptibility tests; approved standard - tenth edition (CLSI document M02-A10). Wayne, PA: Clinical and Laboratory Standards Institute; 2011.

14. Sievert DM, Ricks P, Edwards JR, Schneider A, Patel J, Srinivasan A, et al. Antimicrobial-resistant pathogens 
associated with healthcare-associated infections: summary of data reported to the National Healthcare Safety Network at the Centers for Disease Control and Prevention, 2009-2010. Infection Control Hospital Epidemiology. 2013;34(1):1-14.

15. Moraes GM, Cohrs FM, Batista REA, Grinbaum RS. Infecção ou colonização por micro-organismos resistentes: identificação de preditores. Acta Paul Enferm. 2013;26(2):185-91.

16. Abreu PM, Farias PG, Paiva GS, Almeida AM, Morais PV. Persistence of microbial communities including Pseudomonas aeruginosa in a hospital environment: a potential health hazard. BMC Microbiol. 2014;18:114-8.

17. Ribas RM, Contijo Filho PG, Cezário RC, Silva PF, Langoni DR, Duque AS. Fatores de risco para a colonização por bactérias hospitalares multirresistentes em pacientes críticos, cirúrgicos e clínicos em um hospital universitário brasileiro. Rev Med Minas Gerais. 2009;19(3):193-7.

18. Oliveira AC, Silva RS, Piscoya Díaz ME, Iquiapaza RA. Resistência bacteriana e mortalidade em um centro de terapia intensiva adulto de um Hospital Universitário. Rev Latino-Am Enfermagem. 2010;18(6):1152-60.

19. Oliveira AC, Damasceno QS, Piscoya M, Nicoli JR. Epidemiologic characteristics of resistant microorganisms present in reserves from an intensive care unit. Am J Infect Control. 2012;40(2):1-3.
20. Grice EA, Kong HH, Conlan S, Deming CB, Davis J, Young AC. Topographical and temporal diversity of the human skin microbiome. Science. 2009;324(5931):1190-2.

21. Damaceno Q, Nicolli JR, Oliveira A. Variability of cutaneous and nasal population levels between patients colonized and infected by multidrug-resistant bacteria in two Brazilian intensive care units. SAGE Open Med. 2015;3:1-7.

22. Fortaleza CMB, Freitas FM, Lauterbach GP. Colonization pressure and risk factors for acquisition of imipenem-resistant Acinetobacter baumannii in a medical surgical intensive care unit in Brazil. Am J Infect Control. 2013;41(3):263-5.

23. Almeida GCM, Lima NGM, Santos MM, Melo MCN, Lima KC. Colonizaçấo nasal por Staphylococcus sp. em pacientes internados. Acta Paul Enferm. 2014;27(3):273-9.

24. Kim YJ, Kim S, Kim YR, Lee JY, Park YJ, Kang MW. Risk factors for vancomycin-resistant enterococci infection and mortality in colonized patients on intensive care unit admission. Am J Infect Control. 2012;40(10):1018-9.

25. Babady NE, Gilhuley K, Cianciminio-Bordelon D, Tang YW. Performance characteristics of the Cepheid Xpert vanA Assay for rapid identification of patients at high risk for carriage of vancomycin-resistant Enterococci. J Clin Microbiol. 2012;50(11):3659-63.

26. Mendes W, Pavão ALB, Martins M, Moura ALO, Travassos C. Características de eventos adversos evitáveis em hospitais do Rio de Janeiro. AMB Rev Assoc Med Bras. 2013;59(5):421-8.

\section{Como citar este artigo:}

Arcanjo R, Oliveira AC. Fatores associados à colonização axilar por microrganismos resistentes em pacientes na Unidade de Terapia Intensiva. Rev. Aten. Saúde. 2017;15(51)11-17. 